\title{
Real Space Analysis of Excitonic Interactions and Coherence Length in Helical Aggregates ${ }^{\dagger}$
}

\author{
Andrei Piryatinski, ${ }^{\ddagger}$ Sanford A. Asher ${ }^{\S}$ and Shaul Mukamel ${ }^{*, \sharp}$ \\ Department of Chemistry, University of Rochester, Rochester, New York 14627 and Department of Chemistry, \\ University of Pittsburgh, Pittsburgh, Pennsylvania 15260
}

Received: July 24, 2001; In Final Form: January 15, 2002

\begin{abstract}
The effective exciton coherence length in molecular aggregates is calculated using a nonlocal, real-space representation of the linear optical response. Simulations of polarized absorption and linear dichroism demonstrate that the spectra depend on local properties of the molecular aggregate segment where the exciton coherence is delocalized. This allows us to limit the calculations to small segments of a large aggregate, avoiding the complexity of computing long-range coherences.
\end{abstract}

\section{Introduction}

The exciton states in a perfect crystal represent standing waves of electronic excitation delocalized over the entire crystal $^{1,2}$ with infinitely long coherence lengths. However, this infinite coherence length has little physical significance. In any realistic system such as molecular aggregates, proteins, and polypeptides $^{3}$ we do not expect distant regions to interact coherently. Static disorder and dynamic environmental and structural fluctuations destroy correlations between the phases of distant molecular sites. Typical electronic and vibrational properties are therefore local and should be described by wave packets of excition states. ${ }^{4}$ An intuitive physical picture should then be based on properties of these wave packets rather than on individual exciton eigenstates, which merely provide a convenient basis set for representing the wave packets. Since different spectroscopic techniques prepare different types of wave packets, an excitonically coupled system can display a variety of coherence lengths. This issue was addressed recently for photosynthetic antenna complexes and chromophore aggregates, where the coherence lengths associated with $\mathrm{CD}$, cooperative spontaneous emission (superradiance), and pumpprobe spectroscopy were defined and calculated..$^{5-13}$

The coherence length is crucial for theoretical modeling. If the coherence lengths are very short, any description based on the highly delocalized exciton basis states does not provide an intuitive physical insight, since the long-range coherences of individual exciton states will cancel out once the optical properties are calculated. A local (real space) description of the exciton states is then more adequate for computing exciton coherence lengths. Such description, as proposed below, makes it possible to perform calculations on small segments of molecular aggregates and avoid the complexities of computing unnecessarily long-range coherences.

The presence of two or more molecules, with no parallel transition dipole moments, in a unit cell of an ideal molecular crystal or in repeat unit (RU) of an ideal molecular aggregate causes the Davydov splitting of the absorption exciton band. ${ }^{2}$ In this case, each exciton band can be characterized by its central

\footnotetext{
† Part of the special issue "Mitsuo Tasumi Festschrift".

* Corresponding author. Phone: 716-275-3080. Fax: 716-473-6889. E-mail: mukamel@chem.rochester.edu.

University of Rochester.

$\S$ University of Pittsburgh.
}

(resonance) energy and polarization associated with the orientations of the exciton transition dipoles. For instance, absorption spectra from a molecular crystal or ideal aggregate with two identical nonparallel chromophores in a RU show two perpendicularly polarized absorption bands. ${ }^{2}$ A more interesting example is an infinite molecular aggregate with a helical geometry, whose excitonic absorption spectrum has two bands, polarized parallel and perpendicular to the helical axis. ${ }^{14}$ An important example of such a helical chromophore is the $\alpha$-helix structure in proteins and polypeptides. ${ }^{15}$ Excitonic interactions have a profound influence on the linear and nonlinear optical response in the UV ${ }^{14,16-23}$ and the IR. ${ }^{24-28}$

The Davydov splitting is closely related to the translational symmetry of ideal crystals and is obtained in long molecular aggregates by imposing artificial periodic boundary conditions. If the coherence length associated with the optically produced exciton is short, the polarized spectra should be insensitive to translational symmetry and should rather reflect the local geometry within the exciton coherence length. This can be detected using polarized spectroscopic techniques such as linear dichroism (LD).

This article develops a local real-space picture of optical response of excitons. Our analysis applies to a broad class of dynamical processes involving the electronic excitation in molecular crystals ${ }^{2,29,30}$ and nanostructures ${ }^{4-13,28}$ as well as vibrational response due to interacting localized vibrations, ${ }^{24-27,31}$ which can be described by the Frenkel model; we shall refer to these various types of molecular assemblies as molecular aggregates.

In section II we focus on the coherence lengths associated with polarized linear absorption measurements by introducing the real space picture for the linear Frenkel excitonic response and the corresponding inverse participation ratio (IPR). In section III we present numerical simulations for a model aggregate possessing helical geometry. The linear dichroism ratio and effective polarization angle of exciton wave packets are introduced and calculated in section IV. In section V we discuss our results and conclude.

\section{Exciton Coherence Length}

The ground state $|0\rangle$ of a molecular aggregate with $\mathrm{N}$ units can be written as a direct product 


$$
|0\rangle=\prod_{l=1}^{N}\left|l_{0}\right\rangle
$$

where $\left|l_{0}\right\rangle$ denotes the ground state of the $l$ th unit. The $N$ locally excited configurations $|n\rangle$, whereby only a single RU $n$ is in the excited state $\left|n_{\mathrm{f}}\right\rangle$ form the basis for the single exciton manifold

$$
|n\rangle=\left|n_{\mathrm{f}}\right\rangle \prod_{l \neq n}^{N}\left|l_{0}\right\rangle
$$

where $n=1,2, \ldots N$. These $|n\rangle$ locally excited states are degenerate for the aggregate consisting of identical RU. ${ }^{29,32}$

The excitonic Hamiltonian of the aggregate has the following matrix elements in the local basis set (eq 2)

$$
h_{n m} \equiv\langle n|H| m\rangle=\epsilon_{n} \delta_{n m}+J_{n m}
$$

where $\epsilon_{n}$ is the first excited-state energy of the $n$th unit, renormalized by the Coulombic interaction with the others in their ground states. $J_{n m}$ represents the resonance Coulombic interaction between different locally excited states $|m\rangle$ and $|n\rangle$ and is responsible for exciton splitting. $J_{n m}$ is usually calculated using the dipole-dipole approximation ${ }^{2,25,33-36}$

$$
J_{n m}=\left(\boldsymbol{d}_{\boldsymbol{n}} \boldsymbol{d}_{\boldsymbol{m}}\right) R_{n m}{ }^{-3}-3\left(\boldsymbol{d}_{\boldsymbol{n}} \boldsymbol{R}_{\boldsymbol{n} \boldsymbol{m}}\right)\left(\boldsymbol{d}_{\boldsymbol{m}} \boldsymbol{R}_{\boldsymbol{n} \boldsymbol{m}}\right) R_{n m}{ }^{-5}
$$

where $\boldsymbol{d}_{n}$ and $\boldsymbol{d}_{\boldsymbol{m}}$ are the dipole transition moments of the individual $n$ and $m \mathrm{RU}$, and $\boldsymbol{R}_{\boldsymbol{n} m}$ is the vector connecting the centers of their transition moments. However, the Frenkel exciton model can be applied in a more general case provided charge transfer between adjacent RUs is negligible. In this case, $J_{n m}$ can be calculated by a summation of electrostatic charge pair interaction terms. This requires knowledge of the charge distribution within the chromophores forming the aggregates RU which can be obtained from quantum chemistry calculations. $^{27}$

The stationary (exciton) eigenstates $|k\rangle,(k=1,2, \ldots, N)$ of the Hamiltonian [eq 3] with energies $\omega_{k}$ are linear combinations of the locally excited states $|n\rangle_{.}^{2,28,31}$

$$
|k\rangle=\sum_{n=1}^{N} c_{k n}|n\rangle
$$

We consider the linear absorption spectrum

$$
A(\omega)=\sum_{k}\left|M_{k}\right|^{2} S\left(\omega-\omega_{k}\right)
$$

where $S\left(\omega-\omega_{k}\right)$ is a line shape function, centered at $\omega_{\mathrm{k}}$, and normalized to a unit area. The width of each exciton absorption line is determined by the dephasing rate $\sigma_{k} \cdot M_{k}=\left(\mathrm{M}_{k} \cdot \hat{\mathbf{e}}\right)$, where $\mathbf{M}_{\mathrm{k}}$ is the exciton transition dipole moment and $\hat{\mathbf{e}}$ is a unit vector along the polarization direction of the light. The transition dipoles are

$$
\boldsymbol{M}_{\boldsymbol{k}}=\sum_{n=1}^{N} c_{k n} \boldsymbol{d}_{n}
$$

where $\boldsymbol{d}_{n}$ is the electronic transition dipole of the $n$th RU and $c_{k n}$ is the $k$ th exciton state expansion coefficient [eq 5]. Substituting eq 7 into eq 6 we obtain for the parallel perpendicular and depolarized absorption components

$$
A_{j}(\omega)=\sum_{n m} \alpha_{n m}^{j}(\omega) \quad(j=\|, \perp, d)
$$

where

$$
\alpha_{n m}^{j}(\omega)=\zeta_{n m}^{j} \sum_{k} c_{k n} c_{k m}^{*} S\left(\omega-\omega_{k}\right)
$$

and the polarization prefactors are $\xi_{n m}^{\| l}=\left(\boldsymbol{d}_{n} \hat{\mathbf{e}}^{\prime l}\right)\left(\boldsymbol{d}_{m} \hat{\mathbf{e}}^{\prime \prime}\right), \xi_{n m}^{\perp}=$ $\left(\boldsymbol{d}_{n} \hat{\mathbf{e}}^{\perp}\right)\left(\boldsymbol{d}_{m} \hat{\mathbf{e}}^{\perp}\right)$, and $\xi_{n m}^{d}=1 / 3\left(d_{n}^{x} d_{m}^{x}+d_{n}^{y} d_{m}^{y}+d_{n}^{z} d_{m}^{z}\right)$. Parallel $\hat{\mathbf{e}}^{\|}$ and perpendicular $\hat{\mathbf{e}}^{\perp}$ polarization components are defined with respect to the aggregate axis.

Using eqs 8 and 9 the linear absorption is directly related to the exciton coherence length at energy $\omega$, determined by the variation of $\alpha_{n m}^{a}(\omega)$ with $n-m$. The effective coherence length can be defined by the inverse participation ratio (IPR) associated with $\alpha_{n m}^{a}(\omega)^{10,37,38}$ [Equation 12 in ref 10 for $L_{f}$ can be obtained be substituting $\alpha^{a}{ }_{n m}$ with the exciton Green's function absolute value $\left|G_{n m}\right|$. In this case $L_{f}$ spectral distribution with Lorentzian function for $G_{n m}$ has a width two times smaller than that with $\alpha_{n m}=\operatorname{Im}\left(G_{n m}\right)$. Equation 12 in ref 10 missed a $G_{n m}$ factor in the numerator. Here we give the correct definition, which was used in the calculations reported in ref 10.]

$$
L_{f}^{a}(\omega) \equiv \frac{\left(\sum_{m n} \alpha_{m n}^{a}(\omega)\right)^{2}}{N \sum_{m n}\left(\alpha_{m n}^{a}(\omega)\right)^{2}} \quad(a=\|, \perp, d)
$$

To determine the variation range for the IPR and illustrate its physical significance we consider the following limiting cases.

Case 1. Narrow line width, $\sigma_{k} \ll\left|\omega_{k}-\omega_{k^{\prime}}\right|$, for all $k, k^{\prime}=$ $1, \ldots, N$. In this case the optical excitation at $\omega=\omega_{k}$ can select only a single exciton state corresponding to the following not vanishing term in the sum of eq 9

$$
\alpha_{n m}^{a}\left(\omega_{k}\right) \approx \frac{\xi_{n m}^{a}}{\sigma_{k}} c_{k n} c_{k m}^{*}
$$

where we substitute $S(0)=1 / \sigma_{k}$ for the normalized line shape. The substitution of eq 11 into eq 8 shows that the exciton transitions are well resolved. Normalization of the exciton wave functions gives $c_{k n}(\omega) \propto 1 / \sqrt{N}$. Thus, $\alpha_{n m}^{a}(\omega)$ scales as $\alpha_{n m}^{a}(\omega)$ $\propto 1 / N$ and $L_{f}^{a}=N$. This reflects a uniform (delocalized) distribution of the excitation over the entire aggregate.

Case 2. Broad line width, $\sigma_{k} \gg\left|\omega_{k}-\omega_{k^{\prime}}\right|$. The exciton bands overlap and we get

$$
\alpha_{n m}^{a}(\omega) \approx \frac{\xi_{n m}^{a}}{\sigma} \sum_{k} c_{k n} c_{k m}^{*}=\frac{\xi_{n m}^{a}}{\sigma} \delta_{n m}
$$

$\alpha_{n m}^{a}(\omega)$ is now independent of $N$ and scales as $\alpha_{n m}^{a}(\omega) \propto 1$; eq 10 gives $L_{f}^{a}=1$, indicating that the effective coherence length is equal to unity. This represents the complete localization of the electronic excitation on a single RU. For intermediate values of $\sigma_{k}, L_{f}^{a}(\omega)$ varies between 1 and $N$, providing a precise measure of the relevant exciton size at frequency $\omega$. In the next section we calculate $L_{f}$ numerically for a specific model of the aggregate geometry.

\section{Model Calculations of the Exciton Coherence Length}

We consider an aggregate with $N=100 \mathrm{RU}$ resembling the $\alpha$ helix of protein with 3.6 RU per turn. The helix has a pitch 
(A)

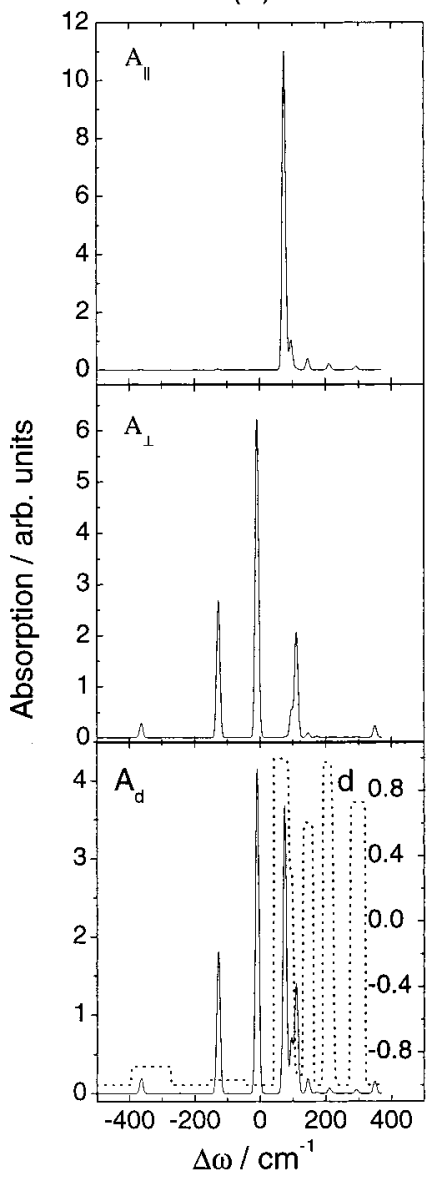

(B)

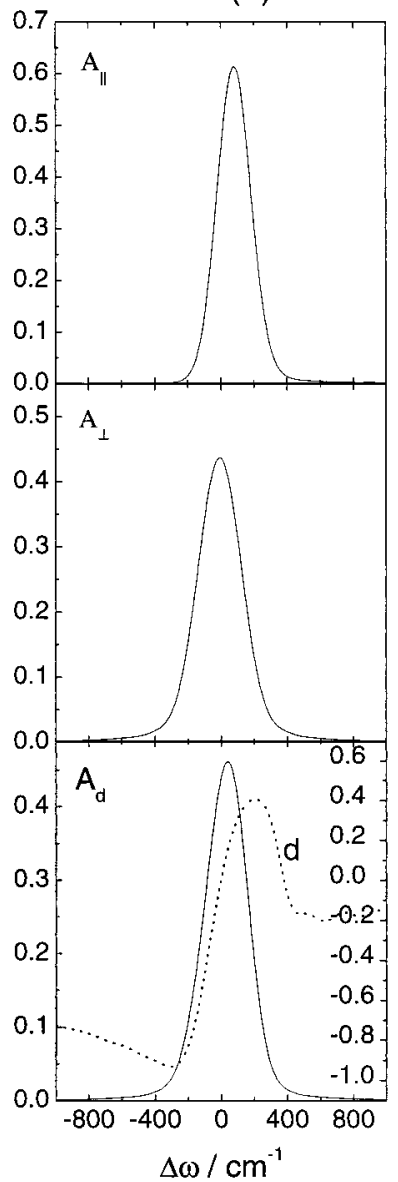

(C)

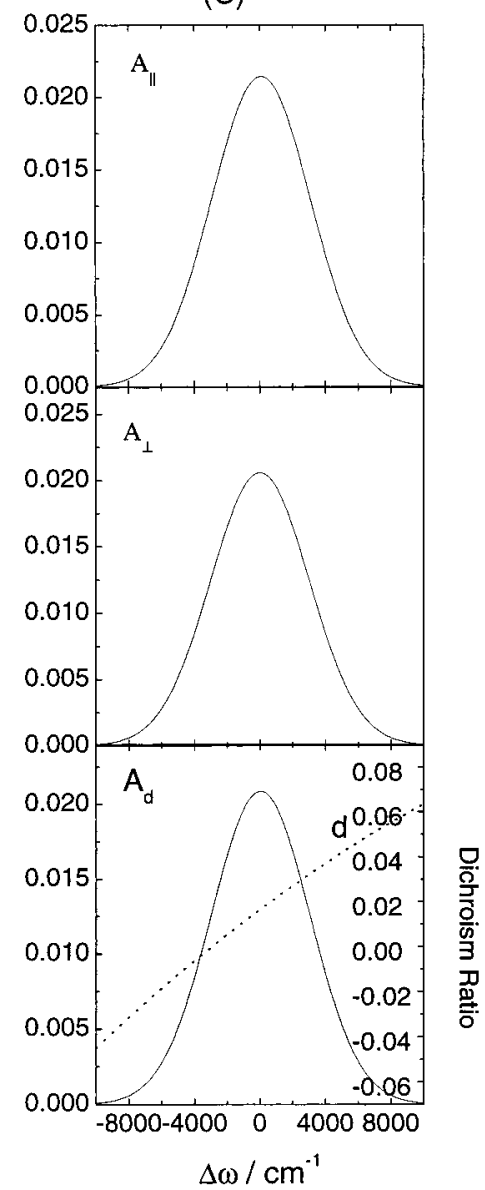

Figure 1. Absorption spectra (solid lines) and linear dichroism (eq 13) (dotted lines) of model helical aggregate consisting of $N=100$ RU, calculated for models $(\mathrm{A})-(\mathrm{C}) . \mathrm{A}_{\| \mid}$and $\mathrm{A}_{\perp}$ are the absorption spectra for light polarized parallel and perpendicular to the oriented helixes, respectively, and $A_{d}$ are depolarized absorption spectra. The origin of the exciton energies is shifted to the center of the depolarized absorption band in (C). (This convention is used though out this paper.)

of $\sim 5.4 \AA$ and the $\mathrm{C}^{\prime}$ carbon atoms lie on a cylindrical surface with a radius of $1.6 \AA$ from the helix axis. Each peptide plane is inclined by $\sim 19^{\circ}$ relative to the helix axis, and the angle between a $\mathrm{C}=\mathrm{O}$ bond and the helix axis is $\sim 22^{\circ}$. Transition moments $\boldsymbol{d}_{\boldsymbol{n}}$ are oriented at $-40^{\circ}$ to the carbonyl bond (positive angles are measured toward the $\mathrm{N}$ atom of amide group). ${ }^{21}$ The helix axis occurs along the (0Z)-axis of our coordinate system, and each RU has a transition dipole moment $\boldsymbol{d}_{\boldsymbol{n}}$ forming angle of $54.3^{\circ}$ with the helical axis. The resonance dipole-dipole Coulombic energies for four closest RU are calculated using eq 4 and set to $J_{n, n+1}=650 \mathrm{~cm}^{-1}$ (given an oscillator strength of 0.198 equal to that of $\left.\mathrm{NMA}^{21}\right), J_{n, n+2}=-278 \mathrm{~cm}^{-1}, J_{n, n+3}$ $=-394 \mathrm{~cm}^{-1}$, and $J_{n, n+4}=58 \mathrm{~cm}^{-1}$, whereas $n+5$ and higher terms are neglected.

The exciton energies, $\omega_{k}$, and wave functions, $c_{k n}$, are evaluated by direct diagonalization of the Hamiltonian (eq 3 ). We note that the calculation of the exciton states in a helical aggregate is equivalent to a 1D molecular chain with four interacting nearest neighbors.

In the following, we assume a Gaussian line shape $S(\omega-$ $\left.\omega_{k}\right)=1 /\left(\sqrt{2 \pi} \sigma_{k}\right) \exp \left[-\left\{\left(\omega-\omega_{k}\right)^{2}\right\} /\left\{2 \sigma_{k}^{2}\right\}\right]$ in eqs 6 and 9 , where $\omega_{k}$ is the exciton energy, and $\sigma_{k}$ is the dephasing rate, set to be identical $\left(\sigma=\sigma_{k}\right.$ ) for all $k$. Three models with (A) weak $\sigma=5 \mathrm{~cm}^{-1}$, (B) intermediate $\sigma=100 \mathrm{~cm}^{-1}$, and (C) strong $\sigma=3000 \mathrm{~cm}^{-1}$ dephasing rates were used. The helices in the ensemble are oriented along the $(0 Z)$ direction, and the polarization prefactors in eq 9 are $\xi_{n m}^{\| l}=d_{n}^{z} d_{m}^{z}$, $\xi_{n m}^{\perp}=1 / 2\left(d_{n}^{x} d_{m}^{x}+d_{n}^{y} d_{m}^{y}\right)$. The coefficient $1 / 2$ in the $\zeta^{\perp}$ comes from averaging over the rotations of the helices around their axis.

The absorption spectra calculated using eqs 8 and 9 are shown in Figure 1. In all calculations we set the origin of the exciton energy scale at the center of the depolarized absorption band in model (C) (the lowest panel in Figure 1 (C)). The dephasing line width in model (A) is smaller than the exciton splitting. Thus, all exciton transitions are well resolved. There is one strong transition polarized along the $(0 Z)$-axis blue shifted from the origin by $\Delta \omega=75 \mathrm{~cm}^{-1}$ and three perpendicularly polarized transitions displaced from the absorption center by $\Delta \omega=-127$ $\mathrm{cm}^{-1}, \Delta \omega=-9 \mathrm{~cm}^{-1}$ and $\Delta \omega=110 \mathrm{~cm}^{-1}$. The parallel spectrum $A_{\|}$is blue shifted compared to the strongest perpendicular one $\left(A_{\perp}\right)$ by $84 \mathrm{~cm}^{-1}$. These two components represent the parallel and perpendicularly polarized lines which could be observed in an ideal (infinite) helix. ${ }^{14}$ The other two strong components resolved in the perpendicular spectrum must vanish in the case of the infinite aggregate. Further increase of the dephasing rate in models (B) and (C) results in the overlap between the parallel and the perpendicular excitonic transitions. They are not resolved in the depolarized spectra in the lowest row of Figure 1.

As shown in section II, for excitation at frequency $\omega$ the exciton state coherence length can be estimated as the FWHM $\epsilon_{a}$ of $\alpha_{n m}^{a}(a=\|, \perp, d)$ plotted vs $m-n$, which must depend on the value of the line width $\sigma$. Figure 2 displays $\alpha_{n m}^{a}$ vs $m-n$. 
(A)

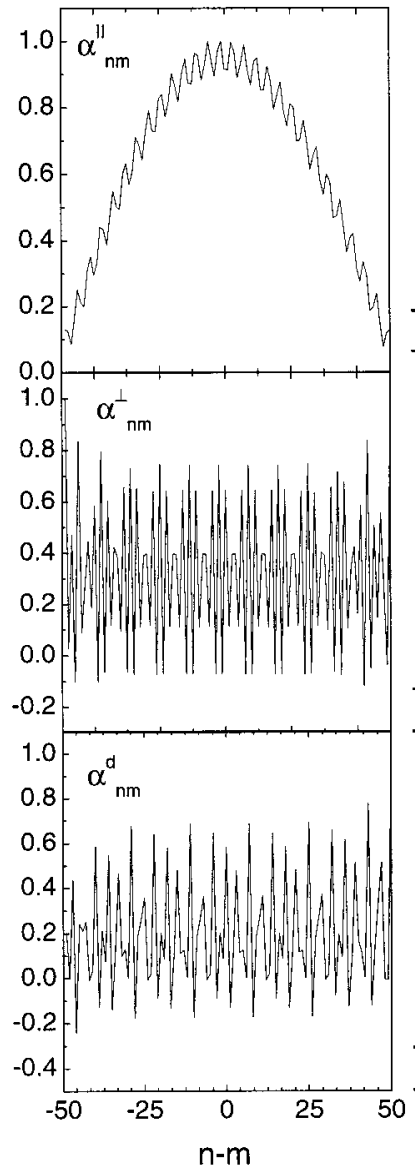

(B)

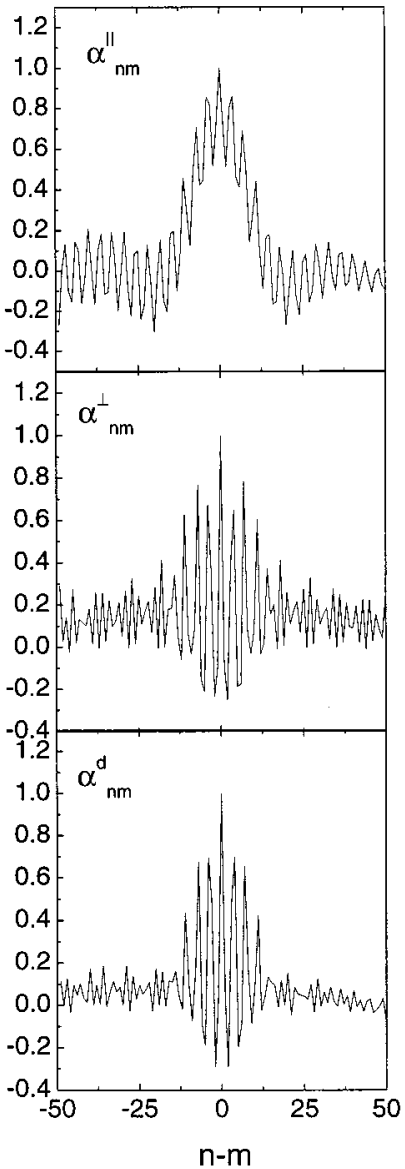

(C)

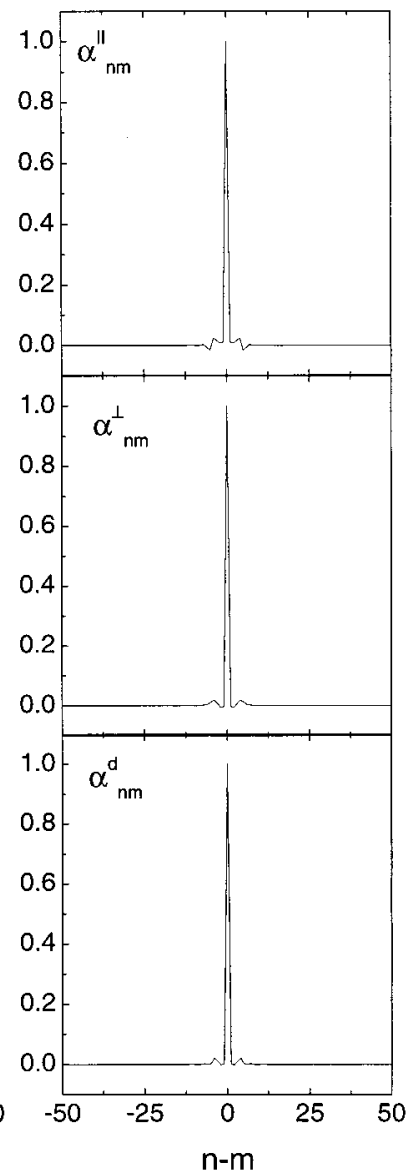

Figure 2. Delocalization of quantities $\alpha_{n m}^{\| \prime} \alpha_{n m}^{\perp}$, and $\alpha_{n m}^{d}$ vs $n-m$ in a helical aggregate consisting of $N=100 \mathrm{RU}$ calculated for models $(\mathrm{A})-(\mathrm{C})$. (A) $\alpha_{n m}^{\prime \prime}$ is calculated at $\Delta \omega=75 \mathrm{~cm}^{-1}$, as well as $\alpha_{n m}^{\perp}$ and $\alpha_{n m}^{d}$ at $\Delta \omega=-9 \mathrm{~cm}^{-1}$. (B) $\alpha_{n m}^{\prime \prime} \alpha_{n m}^{\perp}$ and $\alpha_{n m}^{d}$ are calculated at the centers of the absorption bands $A_{\|}, A_{\perp}$, and $A_{d}$ in Figure $1 \mathrm{~B}$, taking place at $\Delta \omega=78 \mathrm{~cm}^{-1}, \Delta \omega=-7 \mathrm{~cm}^{-1}$, and $\Delta \omega=38 \mathrm{~cm}^{-1}, \mathrm{respectively}(\mathrm{C}) \Delta \omega=0 \mathrm{~cm}^{-1}$ for all $\alpha_{n m}^{\prime \prime} \alpha_{n m}^{\perp}$ and $\alpha_{n m}^{d}$.

Calculations of $\alpha_{n m}^{\|}$and $\alpha_{n m}^{\perp}, \alpha_{n m}^{d}$ in Figure 2A are performed for the strongest exciton transitions that are shifted from the absorption band center by $\Delta \omega=75 \mathrm{~cm}^{-1}$ and $\Delta \omega=-9 \mathrm{~cm}^{-1}$, respectively. The FWHM for this model, $\epsilon_{\|}=70, \epsilon_{\perp}=100$, and $\epsilon_{d}=100$, indicates that the exciton is delocalized over the aggregate. An increase in the exciton line width, results in a gradual decrease in the value of the FWHM as shown in Figures $2 \mathrm{~B}$ and $\mathrm{C}$. For Figure $2 \mathrm{~B}$ it becomes $\epsilon_{\|} \approx \epsilon_{\perp} \approx \epsilon_{d} \approx 20$ at the center of the parallel, perpendicular, and depolarized absorption bands respectively, and for model (C) $\epsilon_{\|}=\epsilon_{\perp}=\epsilon_{d}=1$, indicating that the electronic excitation dephases within 20 (B) and $1(\mathrm{C}) \mathrm{RU}$.

The IPR, depicting the exciton coherence length as the function of the exciton energy, is displayed in Figure 3. For model (A), $L_{f}^{a}(\omega)$ is peaked at the well resolved exciton energies in the absorption spectra (Figure 1). The two main exciton transitions $\left(\Delta \omega=75 \mathrm{~cm}^{-1}\right.$ and $\left.\Delta \omega=-9 \mathrm{~cm}^{-1}\right)$ are delocalized in the polarized spectra, having $L_{f}^{\|}=71$ and $L_{f}^{\perp}=$ 39. The contribution of these transitions to the depolarized absorption spectrum is associated with smaller coherence length $L_{f}^{d}=25$ and $L_{f}^{d}=27$, respectively. As the dephasing rate $\sigma$ is increased in models (B) and (C), the exciton lines overlap, giving rise to smooth behavior of $L_{f}^{a}(\omega)$. To demonstrate that $L_{f}^{a}(\omega)$ is closely correlated with the FWHM of $\alpha_{n m}$, we compare the values of $L_{f}^{a}(\omega)$, given in Figure 3 at the center of each corresponding exciton absorption band, to the width of the associated values of $\epsilon_{a}$ given above. We find the following values of the IPR: (B) $L_{f}^{\|}=16, L_{f}^{\perp}=35, L_{f}^{d}=29$, and (C) $L_{f}^{\|}$ $=L_{f}^{\perp}=L_{f}^{d}=1.2$. These are close to the corresponding $\epsilon_{a}$ values given earlier.

We expect that for a finite exciton coherence length, the absorption spectrum as well as the IPR can be computed using a small segment of an aggregate. This is illustrated in Figure 4, where we show the dependence of $L_{f}^{a}(\omega)$ as well as line shape of $A_{\|}, A_{\perp}$, and $A_{d}$ on the number of $R U$ for the line width adopted in model (C). Panels $1-3$ show the IPR $L_{f}^{a}(\omega)$ for aggregates with different lengths $N=5,10,15,20,25,100$. As $N$ increases, the maxima of the IPR converge to a maximum value of $\sim 1.2$. The difference between the maximum values of $L_{f}^{a}(\omega)$ for $N=5,10,15,20,25$, and $L_{f}^{a}(\omega)$ for $N=100$ is shown in panels $4-6$. For $N=25$ this difference approaches zero and does not change as $N$ grows. In panels $7-9$ we plot the difference between absorption line shape of $N=100 \mathrm{RU}$ aggregate and absorption line shapes of the smaller size $(N=$ $5,10,15,20,25)$ aggregates. All absorption lines were normalized to have the same peak value for $N=25$, the variation in the absorption line shape is of the order of $1 \%$. This suggests that $(\omega)$ and the absorption line shape do not depend on the physical size of the aggregate (provided $N>25$ for the adopted parameters).

We conclude that exciton coherence within the model $\alpha$-helix is delocalized over a small number chromophores for models (B) and (C). The $N=100$ aggregate model is suitable to reproduce delocalized dynamics in the case of the narrow 
(A)

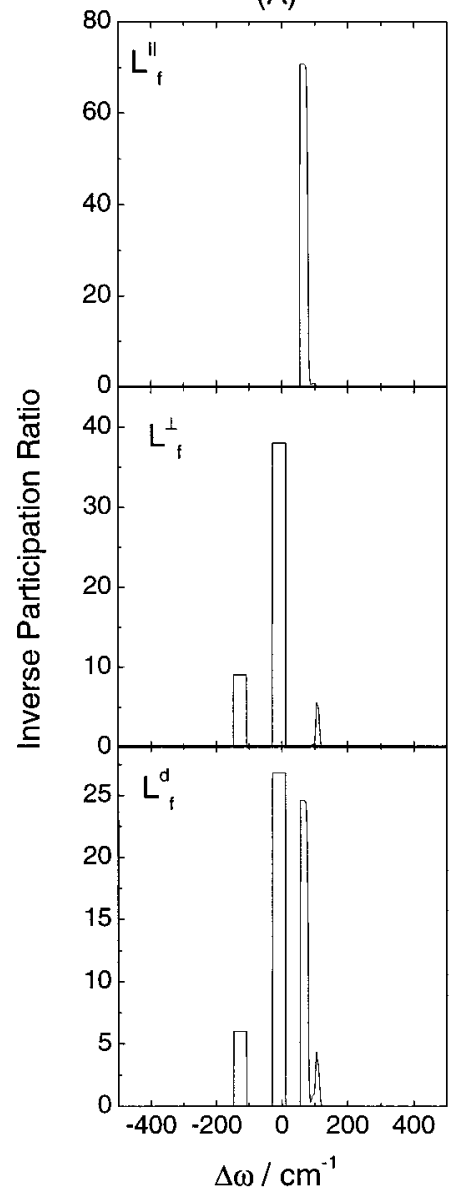

(B)

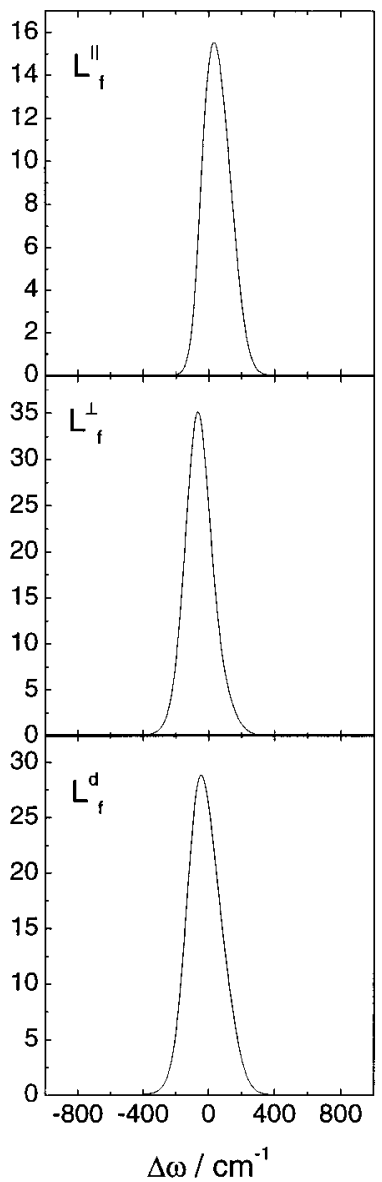

(C)

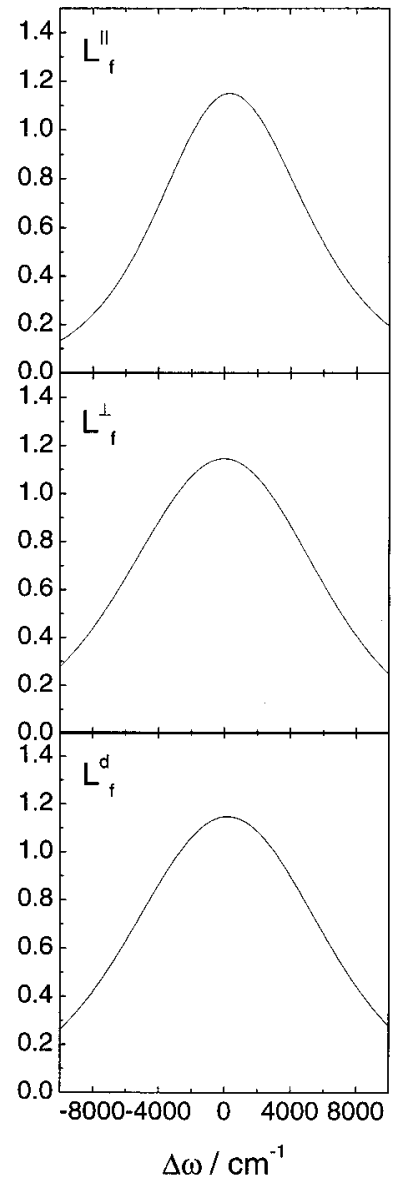

Figure 3. Frequency dependent exciton inverse participation ratio (IPR) $L_{f}^{\|} L_{f}^{\perp}$ and $L_{f}^{d}$ due to optical excitation by light polarized parallel, perpendicular to the (0Z)-axis and by depolarized light, respectively, calculated for models (A) $-(\mathrm{C})$ of a helical aggregate consisting of $N=100$ RU.

absorption line width; however, in addition to the two parallel and perpendicular components predicted for an infinite helix, we see additional well resolved lines in the perpendicular polarized spectrum, resulting from the breakdown of the periodic boundary conditions. The increased overlap between the exciton bands with different polarization in models (B) and (C) leads to a single absorption band with an average polarization. In the next section we investigate this by determining the value of the linear dichroism and the effective polarization angle of the transition dipole.

\section{Polarization of Absorption Spectrum and Exciton Coherence Length}

The linear dichroism (LD) ratio, $d$ is defined as

$$
d \equiv \frac{A_{\|}-A_{\perp}}{A_{\|}+A_{\perp}}
$$

where $d$ assumes the values $-1 \leq d \leq 1$. The value of $d$ indicates whether the parallel or the perpendicular component dominates the absorption spectrum measured with depolarized light.

The LD is plotted for models (A) $-(\mathrm{C})$ of $N=100$, together with the depolarized absorption in Figure 1. For model (A) the LD ratio for the exciton lines at $\Delta \omega=-9 \mathrm{~cm}^{-1}$ and $\Delta \omega=75$ $\mathrm{cm}^{-1}$ is $d=-1$ and $d=1$, indicating that these components are polarized perpendicular and parallel to the helical axis, respectively. The other two components, well resolved in the perpendicularly polarized spectrum at $\Delta \omega=-127 \mathrm{~cm}^{-1}$, and $\Delta \omega=110 \mathrm{~cm}^{-1}$, are $d=-0.97$ and $d=-0.93$, respectively, which are slightly higher than -1 , indicating that these components result from the aggregate finite size. In fact, weak, but still resolved counterparts of these peaks are seen in the upper panel of Figure 1A.

The increased line width [models (B) and (C)] corresponds to stronger overlap between the parallel and perpendicular spectral lines, resulting in the reduction of the range for $d$ to the intervals of $[-0.93,0.4]$ and $[-0.05,0.06]$, respectively. If the broadening and the splitting are of the same order of magnitude as in model (B), the centers of the parallel and perpendicular bands have small offset and this immediately shows up in the LD spectrum as wide variation of the dichroism ratio. Further increase of the dephasing rate in (C) suggests that in the case of large broadening, specific parallel or perpendicular polarization may not be assigned to the exciton wave packets.

The average polarization of the exciton absorption band, with respect to the orientation of the parallel component, at frequency $\omega$ can be defined by the angle $\theta(\omega)$

$$
\cos ^{2}[\theta(\Delta \omega)]=\frac{\sum_{k} \cos ^{2} \theta_{k}\left|\mathbf{M}_{k}\right|^{2} S\left(\Delta \omega-\omega_{k}\right)}{\sum_{k}\left|\mathbf{M}_{k}\right|^{2} S\left(\Delta \omega-\omega_{k}\right)}
$$

where $\theta_{k}$ is the angle between the polarization direction of the 

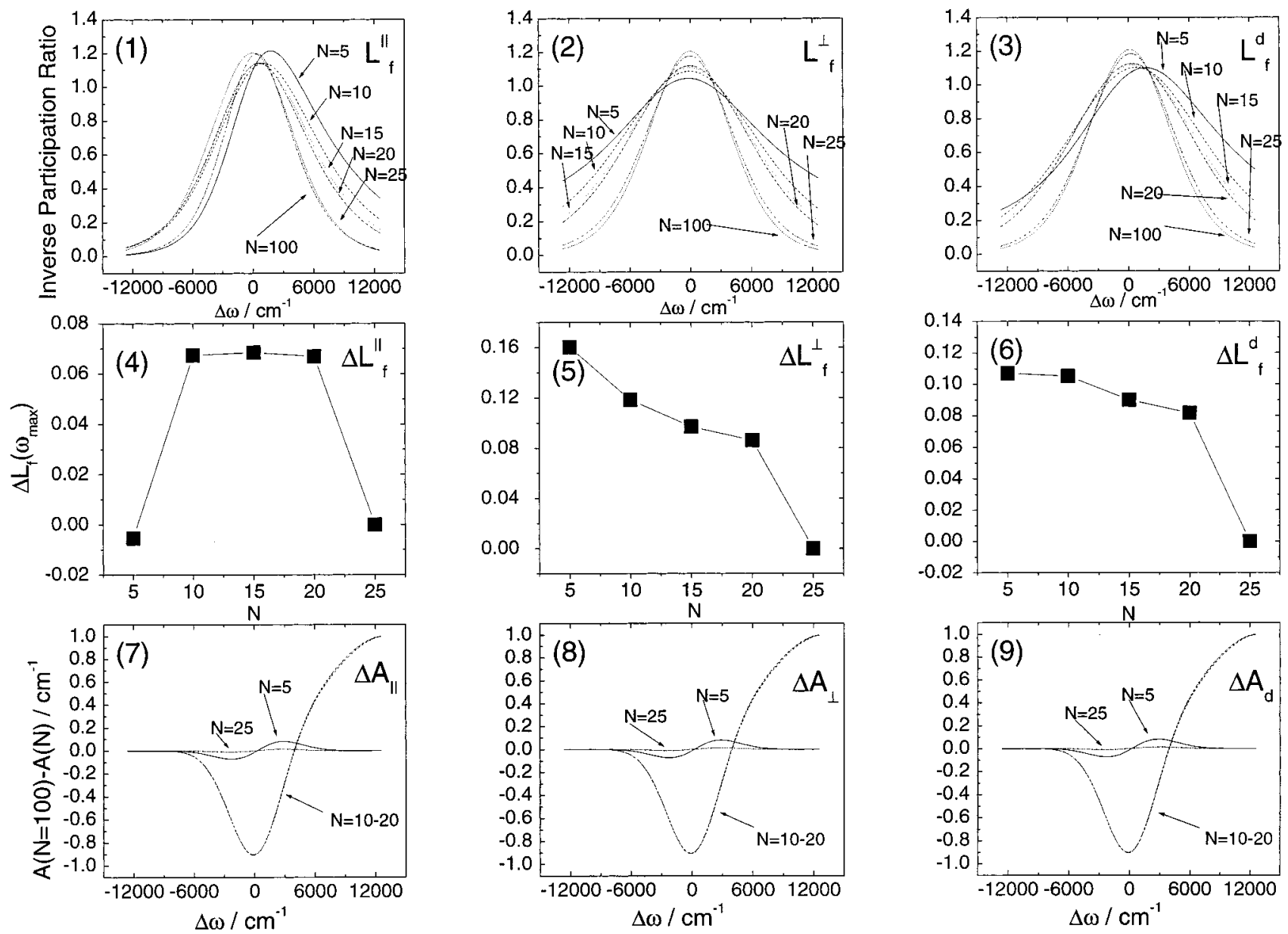

Figure 4. (1-3) Exciton inverse participation ratio (IPR) $L_{f}^{\|} L_{f}^{\perp}$ and $L_{f}^{d}$, calculated for a helical aggregate with $N=5,10,15,20,25,100$ RU. (4-6) Difference between the maximum values of $L_{f}^{\|} L_{f}^{\perp}$ and $L_{f}^{d}$ for $N=5,10,15,20,25$, and 100 chromophores (Figure 3C) of a helical aggregate. (7-9) Difference spectra of $\mathrm{A}_{\|}, \mathrm{A}_{\perp}$, and $\mathrm{A}_{d}$ between $N=100 \mathrm{RU}$ (Figure 1C) and $N=5,10,15,20,25 \mathrm{RU}$ helical aggregates. The dephasing model $(\mathrm{C})$ is adopted in this plot.
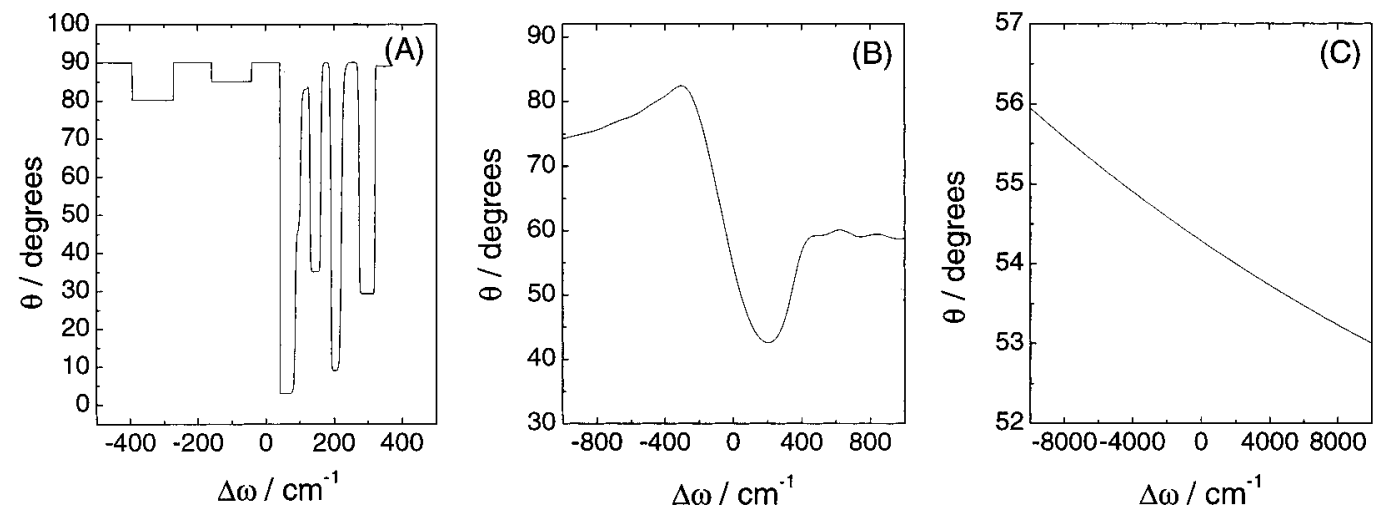

Figure 5. Frequency dependence of $\theta$, the average angle between the excitonic transition dipole and (0Z)-axis for models $(\mathrm{A})-(\mathrm{C})$ of a helical aggregate consisting of $N=100 \mathrm{RU}$.

parallel component of the narrow exciton band and each of the $\boldsymbol{M}_{\boldsymbol{k}}$ transition moments.

The calculated average polarizations for models $(\mathrm{A})-(\mathrm{C})$ are shown in Figure 5. For model (A), eq 14 gives $\theta\left(\omega_{k}\right)=\theta_{k}$. For the exciton transition $\Delta \omega=-9 \mathrm{~cm}^{-1}$ and $\Delta \omega=75 \mathrm{~cm}^{-1}$ the average angle is $\theta=90^{\circ}$ and $\theta=3^{\circ}$, respectively. A decrease in the exciton coherence length due to the increasing value of $\sigma$ in models $(\mathrm{B})$ and $(\mathrm{C})$ dramatically decreases the range of $\theta$. For model (B) $45.6^{\circ} \leq \theta(\Delta \omega) \leq 66.7^{\circ}$, and for model (C) $53.9^{\circ}$ $\leq \theta \leq 54.4^{\circ}$, where $-\sigma \leq \Delta \omega \leq \sigma$. The value of $\theta$ at the center of the depolarized exciton absorption band for models
(B) centered at $\Delta \omega=38 \mathrm{~cm}^{-1}$ is $50.0^{\circ}$, which is close to the $\mathrm{RU}$ polarization angle. For model (C) where the excitation is localized on a single RU the averaged polarization angle at the center of the absorption band $\left(\Delta \omega=0 \mathrm{~cm}^{-1}\right)$ is exactly, $54.3^{\circ}$, which is an RU polarization angle.

\section{Discussion and Conclusions}

The coherence length may be alternatively calculated using a kinematic view based on exciton wave packets and mean free paths. The optical line shapes are broadened either by homo- 
geneous broadening, due, for example, to exciton-phonon coupling or by inhomogeneous broadening, representing, for example, the static disorder of conformations. The incident radiation of frequency $\omega$, therefore, excites several exciton states lying within the bandwidth $2 \sigma$ and creates a wave packet with the momentum spread of $\Delta k,(\Delta k=1, \ldots, N-1$, where $N$ is number of RU). The exciton coherence length would be inversely related to this spread, $L_{f} \equiv N / \Delta k$, reflecting the momentum-position uncertainty principle. Since the exciton energy depends on its momentum $\omega_{k}=\omega(2 \pi k / N)(k=0, \pm$ $1, \ldots, \pm(N-1) / 2, N / 2)$, we find that $2 \sigma \sim \Delta \omega=(\partial \omega(2 \pi k / N) /$ $\partial k) \Delta k$. According to this

$$
L_{f}=\frac{N}{2 \sigma} \frac{\partial \omega(k)}{\partial k}
$$

The dispersion relation ${ }^{30}$ for one-dimensional excitons in the vicinity of an exciton band-edge $(k / N \sim 0)$ is $\omega=4 J \pi^{2} k^{2} / N^{2}$, in the $1 \mathrm{D}$ chain with the nearest neighbor coupling $J$. Substituting this in eq 15 gives an exciton coherence length of $L_{f} \equiv 4 \pi^{2} J k /$ $N \sigma$. Equation 15 can be interpreted as follows: The exciton mean free path is defined as $L_{f}=u \tau$, where $u$ is the exciton wave packet velocity and $\tau$ is the exciton lifetime. Noting that the exciton group velocity is $u=\partial \omega(2 \pi k / N) / \partial(2 \pi k / N)$ and $\sigma=$ $\pi / \tau$ we obtain eq 15 . Equation 15 and our real space approaches lead to identical estimates of the coherence length but provide different physical pictures. Equation 15 is commonly used in molecular crystals, while the approach employed in this paper uses a small local (molecular) basis which is more suitable for molecular aggregates.

In conclusion, using the real space representation for the polarized and depolarized absorption spectra, we have introduced the associated exciton coherence length, which can be related to the exciton mean free path. Our approach, which can be extended to nonlinear coherent techniques, naturally accounts for the specific details of the linear absorption such as the line shape function and the oscillator strengths of the relevant exciton transitions. If the exciton coherence length is small, the absorption spectrum can be computed using a short segment of the aggregate. Consequently, the exciton response reflects local properties such as coupling energies and orientation of the transition dipoles within the molecular aggregate segment where the excitation is delocalized. By comparing the polarization of the exciton bands in an aggregate with the absorption of an ideal aggregate with infinite exciton coherence length as well as with the polarization of the single RU absorption band, one can estimate the exciton localization.

Acknowledgment. The work in Rochester was supported by the National Institutes of Health (GM592030-01A2) and the National Science Foundation (CHE-0132571). This support is gratefully acknowledged. S.A.A. acknowledges the support of NIH grant GM30741.

\section{References and Notes}

(1) Frenkel, J. Phys. Rev. 1931, 37, 17.

(2) Davydov, A. S. Theory of Molecular Excitons; Plenum Press: New York, 1971.

(3) Torii, H.; Tasumi, M. J. Chem. Phys. 1993, 99, 8459.

(4) Chernyak, V.; Wang, N.; Mukamel, S. Phys. Rep. 1995, 263, 213

(5) Grad, J.; Hernandez, G.; Mukamel, S. Phys. Rev. A 1988, 37, 3835.

(6) Spano, F. C.; Mukamel, S. J. Chem. Phys. 1989, 91, 683.

(7) Spano, F. C.; Kuklinski, J. R.; Mukamel S. Phys. Rev. Lett. 1990, $65,211$.

(8) Fidder, H.; Knoester, J.; Wiersma, D. A. Chem. Phys. Lett. 1990 171,529 .

(9) Ozcelic, S.; Akins, D. J. Phys. Chem. 1997, 101, 3021.

(10) Meier, T.; Chernyak, V.; Mukamel, S. J. Phys. Chem. B 1997, 101 , 7332.

(11) Meier, T.; Zhao, Y.; Chernyak, V.; Mukamel, S. J. Chem. Phys. 1997, 107, 1

(12) Zhang, W. M.; Meier, T.; Chernyak, V.; Mukamel, S. Philos. Trans. Royal Soc. A 1998, 356, 405. Zhao, Y.; Meier, T.; Zhang, W. M.; Chernyak, V.; Mukamel, S. J. Phys. Chem. B 1999, 103, 3954.

(13) Dahlbom, M.; Pullerits, T.; Mukamel, S.; Sundstrom, V. J. Phys. Chem. B. 2001, 105, 5515 .

(14) Moffitt, W. J. Chem. Phys. 1956, 25, 467.

(15) Creighton, T. E. Proteins. Structure and Molecular Properties; Freeman: New York, 1993.

(16) Moffitt, W. Proc. Natl. Acad. Sci. U.S.A. 1956, 42, 736.

(17) Woody, R. W. Circular Dichroism and the Conformational Analysis of Biomolecules; Fasman, G. D., Ed.; Plenum Press: New York, 1996; pp 25-67 (and references therein).

(18) Brahms, J.; Pilet, H.; Damany, H.; Chandrasekharan, V. Proc. Natl. Acad. Sci. U.S.A. 1968, 60, 1130.

(19) Cantor, C. R.; Schimmel, P. R. Biophysical Chemistry, W. H. Freeman and Company: San Francisco, 1980; Part II, pp 368-370.

(20) Yamaoka, K.; Ueda, K.; Kosako, I. J. Am. Chem. Soc. 1986, 108 4619.

(21) Pajcini, V.; Asher, S. A. J. Am. Chem. Soc. 1999, 121, 10942.

(22) Woody, R. W. J. Chem. Phys. 1968, 49, 4797.

(23) Serrano-Andrés, L.; Fülscher, M. P. J. Am. Chem. Soc. 1998, 120, 10912.

(24) Krimm S.; Bandeker, J. J. Adv. Protein Chem. 1986, 38, 181.

(25) Hamm, P.; Lim, M.; Hochstrasser, R. M. J. Phys. Chem. B 1998, 102,6123 .

(26) Scherer, C.; Piryatinski, A.; Mukamel, S. J. Am. Chem. Soc. 2001, $123,3114$.

(27) Piryatinski, A.; Chernyak, V.; Mukamel, S. Ultrafast Infrared and Raman Spectroscopy, Fayer, M. D., Ed.; Marcel Dekker: New York, 2001; pp 349-382.

(28) Mukamel, S., Chemla D. S., Eds.; Special issue on Chromophore Aggregates, Chem. Phys. 1996, 210, 1-388.

(29) Agranovich, V. M. Optical Properties of Solids; Abelès, F., Ed.; North-Holland: Amsterdam, 1972; pp 315-350.

(30) Silinsh, E. A.; Chapek, V. Organic Molecular Crystals; American Institute of Physics: New York, 1994.

(31) Mukamel, S. Annu. Rev. Phys. Chem. 2000, 51, 691.

(32) Winston, H. J. Chem. Phys. 1950, 19, 156.

(33) Förster, Th. Delocalized Excitation and Excitation Transfer in Modern Quantum Chemistry, Istanbul Lectures; Sinanogly, O., Ed.; Academic Press: New York, 1965; Part III, pp 93-137.

(34) Tinoco, I., Jr.; Woody, R. W.; Bradley, D. F. J. Chem. Phys. 1963, $38,1317$.

(35) Katsuura, K. J. Chem. Phys. 1964, 40, 3527.

(36) Krueger, B. P.; Scholes, G. D.; Fleming, G. R. J. Phys. Chem. B 1998, 102, 5378

(37) Economou, E. Green Functions Quantum Physics; Springer: New York, 1994

(38) Kirkwood, J. C.; Scheurer, C.; Chernyak, V.; Mukamel, S. J. Chem. Phys. 2000, 114, 2419. 